\title{
SÍNDROME PSEUDO-BARTTER EN PACIENTE PEDIÁTRICO CON FIBROSIS QUÍSTICA ESTABLE
}

\section{PSEUDO-BARTTER'S SYNDROME IN A PEDIATRIC PATIENT \\ WITH STABLE CYSTIC FIBROSIS}

\author{
Dr. Ricardo Madrid H. ${ }^{1}$, Dr Pablo Jorquera P. ${ }^{1}$, Dra Yanina Jaramillo M.. ${ }^{2}$ \\ ${ }_{1}^{1}$ Pediatra Especialista en Enfermedades Respiratorias, Unidad de Broncopulmonar Infantil, Complejo Asistencial Dr. Sótero del Río. \\ 2 Pediatra, Unidad de Emergencia Infantil, Hospital Roberto del Río.
}

\section{ABSTRACT}

The pseudo-Bartter's syndrome (PBS) is a disorder characterized by metabolic alkalosis, hyponatremia, hypochloremia, hypokalemia in the absence of renal tubular disease. The PBS can be one of the complications of cystic fibrosis or may be the initial presentation of the disease in children and adults. The objective is to present a clinical case emphasysing the importance of diagnostic suspicion in cystic fibrosis.

Keywords: cystic fibrosis, pseudo Bartter's syndrome, hypochloremia, metabolic alkalosis

\section{RESUMEN}

El Síndrome de Pseudo-Bartter (SPB)se caracteriza por alcalosis metabólica, hiponatremia, hipocloremia, hipocalemia en ausencia de enfermedad tubular renal. El SPB puede ser una complicación de la Fibrosis Quística (FQ) o la forma de presentación inicial de esta enfermedad, en niños y en adultos. El objetivo es presentar un caso clínico, enfatizando en la importancia de tener un alto índice de sospecha de esta condición.

Palabras clave: fibrosis quística, síndrome pseudo-Bartter, hipocloremia, alcalosis metabólica

\section{CASO CLÍNICO}

Lactante de 8 meses, género femenino, con diagnóstico de $F Q$ sospechada por asma refractaria a tratamiento y neumonía recurrente. El diagnóstico se confirmó por test de sudor (GibsonCooke con cloro $77 \mathrm{mEq} / \mathrm{L})$ y estudio genético $\Delta \mathrm{F} 508 / \mathrm{R} 334 \mathrm{~W}$. La paciente no tenía insuficiencia pancreática, pero presentaba una pérdida de peso de 1000 grs. en los dos últimos meses a pesar del tratamiento estándar para la FQ. Ingresó por escasos vómitos, decaimiento y signos de deshidratación, con aspecto enflaquecida, afebril, gases arteriales y electrolitos plasmáticos mostraron alcalosis metabólica, hipocloremia, hiponatremia e hipokalemia (Tabla 1). Se detectó que no había cumplido la recomendación de ingesta adicional de sal. Después de corregir el trastorno hidroelectrolítico y de la sustitución de sal, la alteración metabólica se corrigió y el incremento de peso fue rápido. No ha vuelto a presentar trastorno metabólico ni hidroelectrolítico.

\section{COMENTARIO}

El SPB puede ser una de las complicaciones de la FQ o puede ser la forma de presentación inicial de la enfermedad, en niños y en adultos $(1,2)$. También se puede presentar

\section{Correspondencia:}

Dr. Ricardo Madrid H.

Unidad de Broncopulmonar Infantil

Complejo Asistencial Dr. Sótero del Río

Av. Concha y Toro 3459, Puente Alto

Santiago, Chile

Correo electrónico: ricardomadridh@gmail.com en neonatos con restricción del crecimiento intrauterino, abuso de laxantes, prematuro hijo de madre con trastornos hidroelectrolítico periparto, diarrea aclorhidrica congénita de la infancia, uso de diuréticos de asa o tiazidicos, enfermedad de Hirschsprung, cualquier causa de hiperemesis aguda grave 0 crónica, enfermedad poliquística renal, uso de gentamicina (3).

Se manifiesta a cualquier edad siendo más común en niños entre los 3 y 14 meses $(4,5)$. En niños mayores de cuatro años es raro observarlo (6). La incidencia de PSB en los pacientes con $\mathrm{FQ}$ es de $12 \%$ a $16.5 \%(1,4,5,7,8)$, y es más frecuente que aparezca en las estaciones del año con mayor temperatura $(9,10)$. Varias de las mutaciones del gen CFTR se

\begin{tabular}{|l|c|c|c|}
\hline & Día 1 & Día 2 & Pre-alta \\
\hline $\mathrm{Na}(\mathrm{mEq} / \mathrm{L})$ & 124 & 138 & 138 \\
\hline $\mathrm{K}(\mathrm{mEq} / \mathrm{L})$ & 2,9 & 4 & 3,7 \\
\hline $\mathrm{Cl}(\mathrm{mEq} / \mathrm{L})$ & 68 & 105 & 110 \\
\hline $\mathrm{pH}$ & 7,64 & 7,47 & 7,5 \\
\hline $\mathrm{HCO3}$ & 42 & 21,7 & 17 \\
\hline $\mathrm{PaCO}_{2}(\mathrm{mmHg})$ & 40 & 31 & 23 \\
\hline B.E. & 19,1 & -1 & -4 \\
\hline
\end{tabular}

Tabla 1. Exámenes de laboratorio

Na: sodio; K: potasio; Cl: cloro; HCO3: bicarbonato; pH: pH de sangre arterial; $\mathrm{PaCO}_{2}$ : presión parcial de dióxido de carbono; $\mathrm{BE}$ : exceso de base; $\mathrm{mEq} / \mathrm{L}$ : miliequivalentes por litro; $\mathrm{mmHg}$ : milímetros de mercurio 
han visto asociadas a deshidratación, hiponatremía y alcalosis metabólica (9) siendo la mutación delta F508 la implicada con mayor frecuencia (3).

Toda situación que se acompaña por aumento de pérdidas de sodio y cloro, se considera un factor de riesgo, como el clima cálido, sudoración profusa, enfermedad gastro intestinal, exacerbación respiratoria, alimentación exclusiva con leche materna, debido a su bajo contenido de sal $(1,11)$. La sudoración en los pacientes con FQ está asociada con un aumento de la concentración de sodio en sudor en aproximadamente 30 $\mathrm{mmol} / \mathrm{L}$ (12). Los pacientes con FQ subestiman sus necesidades de líquidos y electrolitos, experimentado deshidratación durante la exposición a climas cálidos y durante la actividad física intensa (12). La pérdida excesiva de sodio, cloro y agua a través del sudor, llevan a disminución del volumen extracelular, estimulando el sistema renina-angiotensina-aldosterona, la hormona antidiurética y el sistema simpático, lo que genera un aldosteronismo secundario y, por lo tanto un aumento de la reabsorción de sodio, cloro y agua en el tubo colector y excreción de potasio dando lugar a la alcalosis metabólica hipocloremica hipokalemica $(3,9)$.

En los pacientes con FQ los signos clínicos de deshidratación como palidez, llene capilar enlentecido, turgencia anormal de la piel, ausencia de lágrimas, sequedad de mucosas, ojos hundidos y taquicardia, son menos evidentes que los sujetos normales $(12,13)$. El PBS puede presentarse de forma aguda siendo muy sintomático con episodios de vómitos, la forma crónica suele ser menos llamativa y sus síntomas bastante inespecíficos (3). Se han descrito tres patrones de PBS: crisis única y aislada en la infancia, patrón recurrente episódico y patrón crónico (5). El patrón único puede ser la única manifestación de FQ (5). El patrón recurrente episódico es el más común y ocurre en pacientes colonizados por Pseudomona (5).

Como consecuencia del PBS puede aparecer diversas complicaciones $(3,6,10)$ :

- la alcalosis metabólica disminuye las concentraciones de calcio ionizado causando espasmos musculares, convulsiones

- puede presentarse hipoventilación por disminución de los impulsos respiratorios lo que predispone a atelectasias incluso pueden presentar insuficiencia respiratoria grave

- en los pacientes con alcalosis metabólica grave el gasto cardiaco disminuye, la resistencia vascular periférica aumenta y las arritmias cardíacas pueden ser refractarias

- ileo metabólico y agravamiento del PBS

El tratamiento consiste en hidratación y corrección del desequilibrio hidroelectrolítico. La administración de suplemento de electrolitos sodio y potasio debe continuarse hasta que los niveles séricos normalicen $(9,14)$

El diagnóstico diferencial del PBS es: estenosis hipertrofica del píloro, vómitos persistentes, drenaje gástrico continuo sin sustitución adecuada de electrolitos, abuso de laxantes, uso de diuréticos tiazidicos, hiperaldosteronismo primario, síndrome de Bartter, síndrome de Gitelman, diarrea perdedora de cloro $(1,9)$. El PBS se diferencia además del síndrome de Bartter por las altas pérdidas urinarias de cloruro mientras que en el PSB son bajas (11). En los pacientes con displasia broncopulmonar quienes reciben diuréticos de asa 0 tiazidicos puede haber confusión en el diagnóstico diferencial de una alcalosis metabólica (3).

Los niños con $F Q$ que viven en lugares calurosos deben ser suplementados con cloruro de sodio en los momentos de mayores temperaturas (9). A todos los pacientes con FQ se les debe aconsejar evitar la exposición al calor (2). Se recomienda que los recién nacidos con $F Q$ que se alimentan con leche materna o fórmula deben suplementarse con cloruro de sodio $3 \mathrm{mmol} / \mathrm{kg}$, se debe aumentar a $5-6 \mathrm{mmol} / \mathrm{kg}$ bajo circunstancias tales como clima cálido, calefacción excesiva 0 actividad física deportiva (12).

\section{CONCLUSIÓN}

El diagnóstico de $F Q$ debe ser siempre considerado en paciente de cualquier edad con alcalosis metabólica hipoclorémica y deshidratación hiponatrémica asociado o no asociado a compromiso pulmonar o gastrointestinal típico de la $F Q(2,4,14)$. En un paciente con $F Q$ con tratamiento estándar, un mal incremento o pérdida de peso asociado a anorexia debe hacer sospechar PBS especialmente en lactantes en el verano e iniciar su pesquisa y tratamiento para evitar complicaciones.

\section{REFERENCIAS}

1. Vilotijević-Dautović G, Stojanović V. Pseudo-Bartter's Syndrome in Patients with Cystic Fibrosis: A Case Series and Review of the Literature. Srp Arh Celok Lek. 2015;143(11-12):748-51.

2. Priou-Guesdon M, Malinge MC, Augusto JF, Rodien P, Subra JF, Bonneau D, Rohmer V. Hypochloremia and hyponatremia as the initial presentation of cystic fibrosis in three adults. Ann Endocrinol (Paris) 2010;71(1) 46-50.

3. Morales Múnera OL, Villada Valencia JC, Flórez ID, Pineda Trujillo NG. Alcalosis metabólica hipoclorémica como presentación de la fibrosis quística. Informe de dos casos. IATREIA 2013;26(3): 356-365.

4. Maia C, Garrido A, Carvalho I, Pinto H, Guedes Vaz L, Ferreira G. Pseudo-Bartter syndrome as an initial presentation of cystic fibrosis. Port J Nephrol Hypert 2014; 28(4): 348-351.

5. Dahabreh MM, Najada AS. Pseudo-Bartter Syndrome, Pattern and Correlation with Other Cystic Fibrosis Features. Saudi J Kidney Dis Transpl 2013;24(2):292-6

6. Kintu B, Brightwell A. Episodic Seasonal Pseudo-Bartter Syndrome in Cystic Fibrosis. Paediatr Respir Rev 2014;15 Suppl 1:19-21

7. Ballestero Y, Hernandez MI, Rojo P, Manzanares J, Nebreda V, Carbajosa $\mathrm{H}$, Infante E, Baro M. Hyponatremic Dehydration as a Presentation of Cystic Fibrosis. Pediatr Emerg Care. 2006;22(11):725-7

8. Fustik S, Pop-Jordanova N, Slaveska N, Koceva S, Efremov G. Metabolic alkalosis with hypoelectrolytemia in infants with cystic fibrosis. Pediatr Int. 2002;44(3), 289-92.

9. Galaviz-Ballesteros MJ, Acosta-Rodríguez-Bueno CP, ConsueloSánchez A, Franco-Álvarez I, Olalla-Mora OI, Vázquez-Frias R. Síndrome de pseudo-Bartter como presentación de fibrosis quística con mutación DF508 Bol Med Hosp Infant Mex. 2016;73(5):331---334

10. Kose M, Pekcan S, Ozcelik U, Cobanoglu N, Yalcin E, Dogru D, Kiper N. An epidemic of pseudo-Bartter syndrome in cystic fibrosis patients Eur J Pediatr. 2008;167:115-6.

11. Marah MA. Pseudo-Bartter as an initial presentation of cystic fibrosis. A case report and review of the literature. East Mediterr Health $\mathrm{J}$. 2010;16(6):699-701.

12. Scurati-Manzoni E, Fossali EF, Agostoni C, Riva E, Simonetti GD, 
Zanolari-Calderari M, Bianchetti MG, Lava SA. Electrolyte abnormalities in cystic fibrosis: systematic review of the literature. Pediatr Nephrol. 2014;29(6):1015-23.

13. Aranzamendi RJ, Breitman F, Asciutto C, Delgado N, Castaños C. Deshydration and metabolic alkalosis: an unusual presentation of cystic fibroiss in an infant. Arch Argent Pediatr 2008;106(5):443-6.

14. Sovtic A, Minic P, Bogdanovic R, Stajic N, Rodic M, MarkovcSovtic G. Atypical presentation of cystic fibrosis - obese adolescent with hypertension and pseudo-Bartter's syndrome. Vojnosanit Pregl. 2012;69(4): 367-9. 